\title{
Comparison of clinical features between primary and drug-induced sleep-related eating disorder
}

\author{
This article was published in the following Dove Press journal: \\ Neuropsychiatric Disease and Treatment \\ 26 May 2016 \\ Number of times this article has been viewed
}

\author{
Yoko Komada' \\ Yoshikazu Takaesu² \\ Kentaro Matsui ${ }^{3}$ \\ Masaki Nakamura ${ }^{3}$ \\ Shingo Nishida ${ }^{3}$ \\ Meri Kanno ${ }^{3, \dagger}$ \\ Akira Usui ${ }^{3}$ \\ Yuichi Inoue ${ }^{1,3}$ \\ 'Department of Somnology, \\ ${ }^{2}$ Department of Psychiatry, Tokyo \\ Medical University, ${ }^{3}$ Japan Somnology \\ Center, Institute of Neuropsychiatry, \\ Tokyo, Japan \\ †Meri Kanno passed away on \\ March I, 2016
}

Correspondence: Yoko Komada

Department of Somnology, Tokyo

Medical University, 6-I-I Shishinjuku,

Shinjuku-ku, Tokyo 160-8402, Japan

$\mathrm{Tel}+8 \mid 3335$ I 6l4 I

Fax +8I 3 335I 6208

Email ykoma@tokyo-med.ac.jp
Purpose: The aim of this study was to ascertain the clinical characteristics of drug-induced sleep-related eating disorder (SRED).

Patients and methods: We retrospectively reviewed the medical records of 30 patients with primary SRED (without any comorbid sleep disorders and who were not taking any possible causative medications), and ten patients with drug-induced SRED (occurrence of SRED episodes after starting nightly medication of sedative drugs, which completely resolved after dose reduction or discontinuation of the sedatives).

Results: All patients with drug-induced SRED took multiple types of sedatives, such as benzodiazepines or benzodiazepine receptor agonists. Clinical features of drug-induced SRED compared with primary SRED were as follows: higher mean age of onset (40 years old in druginduced SRED vs 26 years old in primary SRED), significantly higher rate of patients who had total amnesia during most of their SRED episodes $(75.0 \%$ vs $31.8 \%)$, significantly lower rate of comorbidity of night eating syndrome ( $0 \%$ vs $63.3 \%)$, and significantly lower rate of history of sleepwalking ( $10.0 \%$ vs $46.7 \%$ ). Increased doses of benzodiazepine receptor agonists may be responsible for drug-induced SRED.

Conclusion: The clinical features of drug-induced SRED were different from those of primary SRED, possibly reflecting differences in the underlying mechanisms between these two categories of SREDs.

Keywords: nocturnal eating syndrome, night eating, eating disorder, hypnotics, amnesia, sleepwalking, benzodiazepine

\section{Introduction}

Sleep-related eating disorder (SRED) is a behavioral disorder in which recurrent episodes of dysfunctional eating after arousal, usually within 2-3 hours of sleep onset, occur almost nightly. ${ }^{1-3}$ Typical SRED frequently features partial loss of consciousness during eating episodes, with subsequent impaired recall. ${ }^{1}$ The prevalence rate of SRED in the general population has been reported to be $1 \%-4.6 \%{ }^{4,5}$ Several reports have shown that SRED often occurs in persons with previous or current episodes of sleepwalking, and the two disorders share some common clinical features. ${ }^{6}$ Both SRED and sleepwalking showed a similar timing of episode particularly during the first half of the night, numerous arousals from stage N3 sleep. ${ }^{6}$ Based on this information, SRED has been considered to occur as a result of a dysfunction in sustaining stable slow-wave sleep, which is similar to sleepwalking. ${ }^{7}$

Night eating syndrome (NES) is another important condition in the disordered nighttime eating spectrum, showing episodes of hyperphagia with full consciousness just before or during the nocturnal sleep period. ${ }^{8}$ Although SRED and NES have been accepted as distinct disease categories, many of their features overlap. ${ }^{9}$ On the other 
hand, there have been numerous reports on SRED related to the use of various psychotropic medications, including hypnotics (particularly benzodiazepines and benzodiazepine receptor agonists [BZDs]), antipsychotics, or their combinations. ${ }^{10-15}$

Recently, we indicated that the prevalence of SRED in psychiatric outpatients was as high as $8.4 \%$, and the hypnosedative effects of the drugs could be responsible for the occurrence of the disorder. ${ }^{16}$ However, to date, similarities and differences in the clinical features of drug-induced SRED and primary SRED (without comorbid sleep disorders and not taking any possible causative medications), such as sex distribution, age of onset, time period of the episodes, level of amnesia, and rate of comorbidity of NES, have not been examined. Identification of differences in these factors may be helpful for establishing a differential diagnosis of these two conditions, as well as for gaining a better understanding of the pathophysiological mechanisms of SRED. We therefore performed this retrospective study to compare clinical features between patients with drug-induced SRED and those with primary SRED.

\section{Patients and methods}

All procedures of the study were conducted in accordance with the guidelines outlined in the Declaration of Helsinki. The protocol of this study was reviewed and approved by the ethics committee of the Neuropsychiatric Research Institute (Tokyo, Japan). Written informed consent for participation was obtained from all the subjects after providing a thorough explanation on the study.

We retrospectively investigated the medical records of patients who visited the outpatient clinic of Japan Somnology Center in Tokyo between May 2003 and April 2011 and who were seeking treatment for eating behaviors during nocturnal sleep periods. A definitive diagnosis of SRED was made for these patients by at least two sleep disorder specialist physicians according to the criteria in the International Classification of Sleep Disorders. ${ }^{1}$ Thirty patients were diagnosed as having primary SRED (without any comorbid sleep disorders, such as restless legs syndrome (RLS) and sleep apnea syndrome (SAS), and were not taking any possible causative medications). There were also ten patients who were diagnosed with drug-induced SRED; they fulfilled the diagnostic criteria of chronic insomnia, and their SRED episodes clearly occurred after starting nightly medication of sedative drugs, and completely resolved after dose reduction or discontinuation of the drugs.

The following demographics and self-reported clinical information of the subjects were analyzed from their medical records: 1) weekly frequency of nocturnal eating behavior episodes; 2) time period during which the episodes mainly occurred (first third, second third, or last third of the nocturnal sleep period); 3 ) the presence or absence of total amnesia during the SRED episodes; 4) the presence or absence of NES-based consensus criteria; ${ }^{8} 5$ ) history of sleepwalking during childhood; 6) Epworth Sleepiness Scale (ESS) score; ${ }^{17}$ and 7) habitual sleep length.

Twenty-seven patients ( 22 of the 30 patients with primary SRED and five of the ten patients with drug-induced SRED) underwent nocturnal polysomnography (n-PSG) using standard systems (Alice 4 and Alice 5; Respironics, Inc., Murrysville, PA, USA). The following recordings were also performed during the n-PSG: 4-channel scalp electroencephalography (C3-A2, C4-A1, O1-A2, and O2-A1), electrooculography, submental electromyography, electrocardiography, measurement of nasal/oral airflow using both a thermistor and nasal pressure sensor, measurement of peripheral oxygen saturation $\left(\mathrm{SpO}_{2}\right)$ using an oximetry sensor, recording of snoring sounds using a microphone, measurement of chest/ abdominal respiratory effort, and electromyography of the anterior tibialis to analyze leg movements (bipolar derivations with two electrodes placed $3 \mathrm{~cm}$ apart on the belly of the anterior tibialis muscle of the right and left legs).

Polysomnograms were scored according to the current criteria for the scoring of sleep and associated events of the American Academy of Sleep Medicine. ${ }^{18}$ Sleep variables were scored as follows: stage N1, N2, N3, and rapid eye movement (REM) sleep ( $\%$ sleep period time), total sleep time, wake after sleep onset, sleep efficiency, arousal index, sleep latency, REM latency, periodic leg movements (PLMs) during sleep index, and apnea-hypopnea index (AHI).

The Student's $t$-test was used to compare continuous variables, and the chi-square test was used to compare categorical variables between primary SRED and drug-induced SRED. All statistical analyses were performed using SPSS Version 11.5 (SPSS Inc., Chicago, IL, USA). A $P$-value $<0.05$ was considered to indicate a statistically significant difference between the two groups.

\section{Results \\ Demographic variables}

The demographics of the 40 individuals with SRED in the current study were as follows: the ratio of women was $62.5 \%$, average age at the first visit was $35.5 \pm 11.2$ (mean \pm SD) years (range: $21-74$ years), mean age at the onset of SRED symptoms was $29.4 \pm 12.6$ years (range: $13-72$ years), mean habitual sleep length was $5.9 \pm 1.3$ hours, and the mean ESS 


\begin{tabular}{|c|c|}
\hline Patients taking three & edications $(n=2)$ \\
\hline $\begin{array}{l}\text { Brotizolam (0.25 mg) } \\
\text { Lormetazepam (1 mg) } \\
\text { Clonazepam (0.5 mg) }\end{array}$ & $\begin{array}{l}\text { Flunitrazepam } \\
\text { Etizolam } \\
\text { Rilmazafone } \\
\text { (unknown dose) }\end{array}$ \\
\hline
\end{tabular}

Patients taking two types of medications $(n=8)$

Triazolam $(0.125 \mathrm{mg}) \quad$ Triazolam $(0.125 \mathrm{mg})$

Zolpidem (10 mg)

Flunitrazepam (1 mg)

Zolpidem (5 mg)

Flunitrazepam (1 mg)

Clonazepam (2 mg)

Flunitrazepam (1 mg)

Bromazepam (6 mg)

Zolpidem (10 mg)

Tri

Triazolam $(0.125 \mathrm{mg})$ Nitrazepam (5 mg)

Nitrazepam (5 mg) Clonazepam (1 mg)

Flunitrazepam (2 mg) Etizolam (1 mg)

Figure I Types and doses of medications used by patients with drug-induced SRED. Abbreviation: SRED, sleep-related eating disorder.

score was $10.2 \pm 5.8$ points. A total of 15 out of the 40 patients $(37.5 \%)$ reported a history of sleepwalking during their childhood. There were five patients with depression, and one patient with schizophrenia as psychiatric comorbidities. ${ }^{19}$ There was one female patient who self-reported probable daytime eating disorder in the past times.

Regarding drug-induced SRED, eight patients (80\%) were taking two types of medications, and the other two patients $(20 \%)$ were taking three types of medications at the time of their first visit. All the patients used BZDs before bedtime, with the exception of clonazepam (Figure 1). The most frequently used medication was flunitrazepam $(n=5)$, followed by zolpidem $(n=3)$ and triazolam $(n=3)$. There were no patients taking antipsychotics at the time of the first visit.

\section{Comparison of descriptive variables between primary and drug-induced SRED}

Table 1 shows the comparison of descriptive variables between primary and drug-induced SRED. There was no significant difference in sex distribution between the two groups $\left(\chi_{(1)}^{2}=0.89, P=0.35\right)$. There were also no significant differences between the two groups in body mass index at the time of the investigation $\left(t_{(37)}=0.56, P=0.58\right)$, self-reported weight gain after the onset of $\operatorname{SRED}\left(t_{(36)}=1.28, P=0.21\right)$, ESS score $\left(t_{(35)}=0.25, P=0.81\right)$, and self-reported habitual sleep length at the investigation $\left(t_{(32)}=0.14, P=0.89\right)$. On the other hand, both age at the time of the investigation and age at the self-reported onset of SRED in the drug-induced SRED group were significantly higher than those of the primary SRED group (age at observation: $t_{(38)}=3.68, P<0.001$; age at the self-reported onset of SRED: $\left.t_{(34)}=3.13, P=0.004\right)$.

There were no significant differences between the two groups in the number of patients who reported having SRED episodes almost every night and during the first third of the sleep period (occurring almost every night: $\chi_{(1)}^{2}=0.083, P=0.77$; occurring during the first third of the sleep period: $\left.\chi_{(1)}^{2}=0.56, P=0.52\right)$. However, the percentage of patients who reported having total amnesia during most of the SRED episodes was significantly higher for

Table I Comparisons of clinical features between patients with SRED and those with drug-induced SRED

\begin{tabular}{|c|c|c|c|c|}
\hline Clinical features & Total $(n=40)$ & Primary SRED $(n=30)$ & Drug-induced SRED $(n=10)$ & $P$-value \\
\hline Female (\%) & 62.5 & 66.7 & 50.0 & 0.35 \\
\hline Age at investigation (years) & $35.5 \pm 11.2$ & $32.2 \pm 0.5$ & $45.3 \pm 15.0$ & $<0.001$ \\
\hline Age at onset (years) & $29.4 \pm 12.6$ & $26.0 \pm 7.4$ & $39.6 \pm 19.0$ & 0.004 \\
\hline Body mass index $\left(\mathrm{kg} / \mathrm{m}^{2}\right)$ & $22.6 \pm 3.4$ & $22.8 \pm 3.5$ & $22.1 \pm 3.5$ & 0.58 \\
\hline Weight gain $(\mathrm{kg})$ & $2.9 \pm 4.8$ & $3.4 \pm 5.0$ & $I . I \pm 3.3$ & 0.21 \\
\hline ESS score & $10.2 \pm 5.8$ & $10.3 \pm 4.6$ & $9.8 \pm 8.5$ & 0.81 \\
\hline Habitual sleep length (hours) & $5.9 \pm 1.3$ & $5.9 \pm 1.2$ & $5.8 \pm 1.5$ & 0.89 \\
\hline $\begin{array}{l}\text { Patients experiencing SRED } \\
\text { episodes almost every night (\%) }\end{array}$ & 51.4 & 50.0 & 40.0 & 0.77 \\
\hline $\begin{array}{l}\text { Patients experiencing SRED } \\
\text { episodes predominantly in the } \\
\text { first third of the sleep period (\%) }\end{array}$ & 86.8 & 89.3 & 80.0 & 0.52 \\
\hline $\begin{array}{l}\text { Patients experiencing episodes } \\
\text { mostly with total amnesia (\%) }\end{array}$ & 43.3 & 31.8 & 75.0 & 0.035 \\
\hline Comorbidity of NES (\%) & 47.5 & 63.3 & 0.0 & 0.001 \\
\hline $\begin{array}{l}\text { History of sleepwalking during } \\
\text { childhood (\%) }\end{array}$ & 37.5 & 46.7 & 10.0 & 0.038 \\
\hline
\end{tabular}

Notes: Age at onset, age at the self-reported onset of SRED; ESS score, Epworth Sleepiness Scale score at the time of the investigation; weight gain, self-reported weight gain after SRED onset. Continuous variables are presented as the mean \pm SD. Data presented as mean \pm SD.

Abbreviations: SRED, sleep-related eating disorder; NES, nocturnal eating syndrome. 
the drug-induced SRED group than for the primary SRED group $\left(\chi_{(1)}^{2}=4.46, P=0.035\right)$. The rate of NES comorbidity was $63.3 \%$ in the primary SRED group, whereas there were no patients with NES comorbidity in the drug-induced SRED group $\left(\chi_{(1)}^{2}=12.1, P=0.001\right)$. A significantly larger fraction of patients with primary SRED had a history of sleepwalking during their childhood than patients with drug-induced SRED $\left(\chi_{(1)}^{2}=4.30, P=0.038\right)$.

\section{Results of polysomnographic evaluation}

Of the 27 patients with SRED who underwent PSG evaluation, ten patients (eight patients with primary SRED and two patients with drug-induced SRED) showed SRED episodes on the night of PSG evaluation. Six patients experienced one episode, two patients experienced two episodes, and the other two patients experienced three episodes. Of the 16 total episodes, one episode occurred during stage N3, 12 episodes during stage N2, and three episodes during the awake stage.

Differences in sleep parameters between the two groups are summarized in Table 2 . There were no significant differences between the groups regarding the amount of time spent in lighter sleep ( $\%$ N1, \% N2), REM sleep (\% REM), total sleep time, wake after sleep onset, sleep efficiency, arousal index, REM latency, PLM index, and AHI. However, there were significant differences between the two groups in the amount of time spent in deep sleep $(\% \mathrm{~N} 3)$ and sleep latency. Drug-induced SRED showed a smaller amount of \% N3 sleep and longer sleep latency.

\section{Discussion}

In the current study, primary SRED showed female predominance, and the age of onset of the disorder was concentrated in early adulthood, from the mid-20s to mid-30s. These characteristics were quite similar to those of previously reported SRED cases. ${ }^{2,20,21}$ The rate of history of sleepwalking during childhood was clearly higher in our primary SRED patients (44\%) than in our drug-induced SRED patients $(10 \%)$, although this rate was lower than those previously reported for primary SRED patients $(66 \%-84 \%))^{2,4,6}$ This finding may suggest that primary SRED patients are often predisposed to arousal disorder, ${ }^{2}$ whereas this disorder is less likely to occur in drug-induced SRED patients.

Interestingly, age at the onset of SRED was significantly higher in drug-induced SRED patients than in primary SRED patients, and there was no female predominance in the former group, that is, drug-induced SRED occurred even in the relatively older population, irrespective of sex. Moreover, total amnesia of the episode was relatively common in the druginduced SRED group. Anterograde amnesia or suppression of the transition from sleep to wakefulness due to the use of BZDs $^{22}$ could explain the mechanism of this phenomenon. Conversely, the comorbidity of NES in patients who were fully aware and could recall the nocturnal eating episode ${ }^{8}$ was not observed in patients with drug-induced SRED.

The rate of NES comorbidity was $63.3 \%$ in the primary SRED group. The eating and drinking behavior of SRED occurs upon transition from sleep to awake, typically with reduced consciousness. On the other hand, patients with NES have episodes of conscious night eating (eating after awakening from sleep). Due to this difference, some critical reviews of the published work on SRED and NES have suggested that these conditions are situated at opposite poles of a spectrum of disordered eating during the main sleep period. ${ }^{23}$ However, recent studies have shown cases where

Table 2 Comparison of polysomnographic variables between patients with primary SRED and those with drug-induced SRED

\begin{tabular}{|c|c|c|c|c|}
\hline Polysomnographic variables & Primary SRED (n=22) & Drug-induced SRED $(n=5)$ & $t$-value & $P$-value \\
\hline$\%$ NI sleep & $10.2 \pm 4.6$ & $7.4 \pm 3.0$ & 1.28 & 0.21 \\
\hline$\%$ N2 sleep & $52.9 \pm 11.8$ & $62.5 \pm 5.7$ & 1.76 & 0.091 \\
\hline$\%$ N3 sleep & $6.8 \pm 5.4$ & $\mathrm{I} .4 \pm \mathrm{I} .3$ & 2.15 & 0.041 \\
\hline \% REM sleep & $18.9 \pm 5.0$ & $15.2 \pm 6.5$ & $\mathrm{I} .43$ & 0.17 \\
\hline TST, minutes & $449.0 \pm 68.2$ & $460.4 \pm 113.5$ & 0.30 & 0.77 \\
\hline$\%$ WASO & $1 \mathrm{I} .1 \pm 10.0$ & $13.4 \pm 12.1$ & 0.45 & 0.66 \\
\hline Sleep efficiency, \% & $86.7 \pm 10.8$ & $82.2 \pm 12.7$ & 0.81 & 0.42 \\
\hline Arousal index & $|4| \pm 5.0$. & $10.9 \pm 2.9$ & 1.37 & 0.18 \\
\hline Sleep latency, minutes & $9.8 \pm 12.5$ & $27.0 \pm 23.1$ & 2.35 & 0.027 \\
\hline REM latency, minutes & $90.9 \pm 72.7$ & $162.3 \pm 155.1$ & 1.58 & 0.13 \\
\hline PLM index & $1.2 \pm 2.8$ & $0.0 \pm 0.0$ & 0.63 & 0.37 \\
\hline $\mathrm{AHI}$ & $2.4 \pm 3.5$ & $1.3 \pm 0.7$ & 0.63 & 0.54 \\
\hline
\end{tabular}

Notes: NI, stage I sleep; N2, stage 2 sleep; N3, deep sleep. Data presented as mean \pm SD.

Abbreviations: AHI, apnea-hypopnea index; PLM, periodic limb movement; REM, rapid eye movement; SRED, sleep-related eating disorder; TST, total sleep time; WASO, wake after sleep onset. 
these two disorders coexist; in addition, there has been some argument about similarities and differences between them. The term "nighttime eating" is commonly used by eating disorder researchers to describe both evening hyperphagia (eating after the evening meal, but prior to the initial sleep onset) and nocturnal eating (eating after an arousal from sleep, but prior to the final morning awakening), while the SRED is used by sleep disorder specialist physicians or researchers to describe amnestic ambulation with eating (sleepwalking-like behavior). Considering this, the researchers of these two fields may use divergent, but occasionally overlapping, terminology that is perplexing and impedes clinical investigation. ${ }^{9}$

In this study, all the patients with drug-induced SRED took multiple types of sedative BZDs. Previous reports have emphasized that zolpidem was strongly associated with the presence of SRED. ${ }^{10,24,25}$ However, in the current study, zolpidem was not the only drug responsible for the occurrence of SRED, and the combined use of various types of BZDs was thought to contribute to the occurrence of SRED. The doses of hypnotics used by patients with druginduced SRED were relatively high, which was similar to a previous study. ${ }^{25}$

The PSG results showed that drug-induced SRED patients showed a longer sleep-onset latency and a smaller amount of deep sleep. This phenomenon is possibly related to their insomnia symptoms or the pharmacological effects of the hypnotics. ${ }^{26}$ Although the number of patients undergoing PSG was not sufficient to draw a conclusion, there appeared to be no clear differences in PSG variables between primary SRED and drug-induced SRED. Reportedly, SRED is more prevalent in both patients with RLS and patients with SAS than in controls. ${ }^{25,27}$ Although the nature of the association between SRED and these disorders and its underlying mechanisms remain unclear, the presence of RLS and SAS may contribute to the occurrence of parasomnia via sleep disruptions and fragmentations. ${ }^{24}$ However, in this study, both the PLM index and AHI were in the normal range in the subject of two groups, because we targeted primary SRED without any comorbid sleep disorders and drug-induced SRED. It has been reported that there is a high rate of comorbidity between SRED and daytime eating disorders. For example, Winkelman ${ }^{2}$ found that $40 \%$ of those with SRED were also diagnosed with an eating disorder. When compared with the previous studies, the rate of comorbidity between SRED and eating disorder among our patients was comparatively low (3.3\%).

This study has several limitations. First, the sample size of patients with SRED, particularly drug-induced SRED, was not large enough to draw any definite conclusions. Future larger-scale studies that replicate our results are necessary. Second, the number of tests was performed at a $P<0.05$ for statistical significance. We should be aware that the rate of type I error is high.

\section{Conclusion}

The use of high doses of BZDs may be responsible for the occurrence of drug-induced SRED. The characteristics of drug-induced SRED were different from those of primary SRED, in that there was no female predominance, age of onset was relatively high, episodes with total amnesia were frequent, and NES comorbidity was rare. These characteristics may be helpful for differentiating these two conditions. It would be recommended to reduce the dose or discontinue the usage of BDZs, in the case of suspected drug-induced SRED having the characteristics noted earlier.

\section{Acknowledgments}

The authors acknowledge the support of Doctor Meri Kanno who passed away on March 1, 2016. She was a valued psychiatrist as well as a loving mother, and she will be dearly missed by her friends and colleagues.

The authors are indebted to the medical editors from the Department of International Medical Communications of Tokyo Medical University for editing and reviewing the English manuscript.

This work was supported by a Grant-in-Aid for Scientific Research from the Japan Society for the Promotion of Science (JSPS KAKENHI Grant Number 22700781).

This article is a follow-up of our previous study titled "Comparison of clinical features between primary and secondary sleep-related eating syndrome", which was presented at the Fifth World Congress on Sleep Medicine in Valencia, Spain, during the period September 28, 2013 to October 2, 2013, as an abstract with interim findings. The abstract was published in a supplement of Sleep Medicine on December 2013 and can be viewed at http://www.sciencedirect.com/ science/article/pii/S1389945713016195.

\section{Disclosure}

Yuichi Inoue has received clinically pertinent fees, lecture fees, and research funding from Nippon Boehringer Ingelheim, Takeda Pharmaceutical Company Limited, Mitsubishi Tanabe Pharma, Astellas Pharma Inc., Shionogi, and Sanofi-Aventis. The authors report no other conflicts of interest in this work. 


\section{References}

1. American Academy of Sleep Medicine, editor. International Classification of Sleep Disorders. 3rd ed. Darien, IL: American Academy of Sleep Medicine; 2014.

2. Winkelman JW. Clinical and polysomnographic features of sleep-related eating disorder. J Clin Psychiatry. 1998;59(1):14-19.

3. Vetrugno R, Manconi M, Ferini-Strambi L, Provini F, Plazzi G, Montagna P. Nocturnal eating: sleep-related eating disorder or night eating syndrome? A videopolysomnographic study. Sleep. 2006;29(7): 949-954.

4. Schenck CH, Hurwitz TD, Bundlie SR, Mahowald MW. Sleep-related eating disorders: polysomnographic correlates of a heterogeneous syndrome distinct from daytime eating disorders. Sleep. 1991;14(5): 419-431.

5. Winkelman JW, Herzog DB, Fava M. The prevalence of sleep-related eating disorder in psychiatric and non-psychiatric populations. Psychol Med. 1999;29(6):1461-1466.

6. Brion A, Flamand M, Oudiette D, Voillery D, Golmard JL, Arnulf I. Sleeprelated eating disorder versus sleepwalking: a controlled study. Sleep Med. 2012;13(8):1094-1101.

7. Zadra A, Pilon M, Montplaisir J. Polysomnographic diagnosis of sleepwalking: effects of sleep deprivation. Ann Neurol. 2008;63(4): 513-519.

8. Allison KC, Lundgren JD, O'Reardon JP, et al. Proposed diagnostic criteria for night eating syndrome. Int J Eat Disord. 2010;43(3):241-247.

9. Winkelman JW, Johnson EA, Richards LM. Sleep-related eating disorder. Handb Clin Neurol. 2011;98:577-585.

10. Morgenthaler TI, Silber MH. Amnestic sleep-related eating disorder associated with zolpidem. Sleep Med. 2002;3(4):323-327.

11. Chiang A, Krystal A. Report of two cases where sleep related eating behavior occurred with the extended-release formulation but not the immediate-release formulation of a sedative-hypnotic agent. $J$ Clin Sleep Med. 2008;4(2):155-156.

12. Dang A, Garg G, Rataboli PV. Zolpidem induced Nocturnal SleepRelated Eating Disorder (NSRED) in a male patient. Int J Eat Disord. 2009;42(4):385-386.

13. Najjar M. Zolpidem and amnestic sleep related eating disorder. J Clin Sleep Med. 2007;3(6):637-638.

14. Paquet V, Strul J, Servais L, Pelc I, Fossion P. Sleep-related eating disorder induced by olanzapine. J Clin Psychiatry. 2002;63(7):597.
15. Lu ML, Shen WW. Sleep-related eating disorder induced by risperidone. J Clin Psychiatry. 2004;65(2):273-274.

16. Takaesu Y, Ishikawa J, Komada Y, et al. Prevalence of and factors associated with sleep-related eating disorder in psychiatric outpatients taking hypnotics. Psychiatry Res. 2015;230(3):958-963.

17. Johns MW. A new method for measuring daytime sleepiness: the Epworth sleepiness scale. Sleep. 1991;14(6):540-545.

18. Iber C, Ancoli-Israel S, Chesson A, Quan SF. The AASM Manual for the Scoring of Sleep and Associated Events: Rules, Terminology, and Technical Specifications. Westchester, IL: American Academy of Sleep Medicine; 2007.

19. AmericanPsychiatricAssociation. Diagnostic and Statistical Manual of Mental Disorders. 5th ed. Washington, DC: American Psychiatric Association; 2013.

20. Schenck CH, Hurwitz TD, O'Connor KA, Mahowald MW. Additional categories of sleep-related eating disorders and the current status of treatment. Sleep. 1993;16(5):457-466.

21. Benca RM, Schenck CH. Sleep and eating disorders. In: Kryger MH, Roth T, Dement WC, editors. Principles and Practice of Sleep Medicine. Philadelphia: Elsevier Saunders; 2005:1337-1344.

22. Mitler MM. Nonselective and selective benzodiazepine receptor agonists - where are we today? Sleep. 2000;23:S39-S47.

23. Howell MJ, Schenck CH, Crow SJ. A review of nighttime eating disorders. Sleep Med Rev. 2009;13(1):23-34.

24. Lam SP, Fong SY, Ho CK, Yu MW, Wing YK. Parasomnia among psychiatric outpatients: a clinical, epidemiologic, cross-sectional study. J Clin Psychiatry. 2008;69(9):1374-1382.

25. Wing YK, Lam SP, Li SX, Zhang J, Yu MW. Sleep-related eating disorder and zolpidem: an open interventional cohort study. J Clin Psychiatry. 2010;71(5):653-656.

26. Wallace DM. Hypnotic medications: mechanisms of action and pharmacologic effects. In: Kryger MH, Roth T, Dement WC, editors. Principles and Practice of Sleep Medicine. Philadelphia: Elsevier Saunders; 2005:444-451.

27. Provini F, Antelmi E, Vignatelli L, et al. Association of restless legs syndrome with nocturnal eating: a case-control study. Mov Disord. 2009; 24(6):871-877.
Neuropsychiatric Disease and Treatment

\section{Publish your work in this journal}

Neuropsychiatric Disease and Treatment is an international, peerreviewed journal of clinical therapeutics and pharmacology focusing on concise rapid reporting of clinical or pre-clinical studies on a range of neuropsychiatric and neurological disorders. This journal is indexed on PubMed Central, the 'PsycINFO' database and CAS,

\section{Dovepress}

and is the official journal of The International Neuropsychiatric Association (INA). The manuscript management system is completely online and includes a very quick and fair peer-review system, which is all easy to use. Visit http://www.dovepress.com/testimonials.php to read real quotes from published authors. 